\title{
Design and Testing of a Wireless Sensor Network Using RFID and Zigbee Indoors for Intelligent Buildings
}

\author{
Roberto Ferro Escobar, Víctor Hugo Medina García, and Giovanny Tarazona Bermúdez
}

\begin{abstract}
One of the topics of study and research that has been most discussed in recent years, it refers to wireless sensor networks (WSN), which are used in many fields such as agronomy, army and tracking disaster, usually the measurements are always performed outdoors. This article presents a design of a wireless sensor network and identification of key parameters in a closed environment, then a hybrid hierarchical architecture between Zigbee and RFID technologies is proposed.

The design was applied in the research building of the District University "Francisco José de Caldas", however, the design can be applied to any building or closed room of the same characteristics.
\end{abstract}

Index Terms-RFID, RSSI, WSN, Zigbee.

\section{INTRODUCTION}

A wireless sensor network can be defined as one consisting of elements of measurement, computing and communication are implemented on a given site infrastructure for this purpose, which can be in open spaces [1], [2] and closed [3], [4] this network allows users to test, monitor and react to events that are detected within that range. Among the applications of WSN networks are security, health, agriculture [5], research, monitoring of environmental variables [6], [7], monitoring [8], smart cities and many others who need fast data acquisition and low implementation cost. Although most sensor networks are open, remote and inaccessible environments, it is also possible the deployment of a network of this kind of closed sites, however the design aspects to consider are different.

This paper defined in the following aspects: In the Section II a review of related works will be carry out measurements in enclosed spaces, the Section III will describe the area of interest to be monitored by the sensor network, the Section IV describe the elements used in the design and implementation, the Section $\mathrm{V}$ will show the testing of communications, the Section VI will show the design of the network of sensors and wireless communication tests performed, the Section VII will show network services and the last Section VIII shows the conclusions of the work done.

\section{STATE OF THE ART}

There have been multiple related wireless sensor networks

Manuscript received July 31, 2015; revised September 5, 2015.

The authors are with the District University Francisco José de Caldas, Bogotá, Colombia (tel.: 3239300 ext. 1711, 1010, 1402; e-mail: rferro@udistrital.edu.co, gtarazona@udistrital.edu.co). work, lean toward the search for improvements in energy consumption deployed in open spaces, where the need to have a durable power supply networks is quite high, because many sometimes the nodes become inaccessible after being deployed.

However, there are also developed on experimental work with closed sensor networks [9] spaces, mainly for monitoring and navigation tasks of devices, with the visibility points sensor nodes deployed within the closed environment. As for the design of a network of sensors in open environments is a priority use items that are efficient in terms of energy consumption [10]-[12] and thus the lifetime of the network is as long as possible, for closed environments becomes the main problem in obtaining correct and clean data obtained via sensors, as losses [13] by reflection and refraction are very high and sensors usually have no direct line of sight, which deploy a network of sensors range is a complicated issue.

The simplest and most commonly used to define the scope of a node within a wireless network technique RSSI measurement is [14], [15] (Received Signal Strength Indicator), defined indifferently as the power or voltage measured by a receiver wireless. At present, virtually any wireless receiver has a RSSI indicator included. The RSSI, given a transmission distance is defined in a follows (1):

$$
\operatorname{RSSI}(d)=P L\left(d_{0}\right)-10 \beta \log _{10}\left(d / d_{0}\right)+\omega
$$

where $d$ is a transmition distance; $d_{0}$ is the reference distance; $\beta$ is the rate at which the signal decreases; $\omega$ is a random variable with normal probability; $\operatorname{PL}\left(d_{0}\right)$ is the received signal strength and $\operatorname{PL}\left(d_{0}\right)$ is the signal power from the transmitter. With RSSI measurement, we can get the maximum communication distance between two nodes in a wireless network; this article uses this technique to define the maximum distance between the distribution nodes deployed. Similarly, as in most deployments of sensor networks, Zigbee communication devices [16] they are used [17], and RFID [18] technology for wireless communication between the nodes and the trace elements Tags RFID and NFC.

\section{DEFINITION OF INTEREST AREA}

Building Research at the District University "Francisco José de Caldas" is part of a project designed and under construction, which will house various academic units and are located in the area known as the "Aduanilla of Paiba". This project will complete its construction in 2016, and in it laboratories and offices for innovation and projects of several research groups that are part of the University will be found. This building is designed to be a sustainable building, which supports the growth of the University in the research and 
cultural fields, so the development of a network of sensors and identification was designed in the same way, for complete integration with the building.

Because the building is still under construction, they have not yet defined what kind of qualitative research labs will be in the space for these activities. However, the design of the sensor network allows any type of sensor to be included and their measurements are stored, views and processed by the system.

\section{NETWORK ELEMENTS}

For the development of the sensor network verification components to be used for measurement, transmission and processing of the variables that are within the area of interest is performed.

Designed sensor network is composed of 3 devices:

- Accumulator measures: This device shall consist of three main parts:

- Micro-controller: This device is responsible for collecting all the values obtained by the sensors, store them in memory and transmit. You must also be capable of accepting scheduling algorithms to handle on and off times of the sensors and transmission.

Due to the battery must allow connection of various sensors, some of which may require high accuracy, and also must perform communication via serial transmission with the Zigbee device and the receiver of RFID, has selected the microcontroller PIC24FV32KA304 Microchip, which with its 20-pin encapsulated node can provide up to 12 ports A / D with a resolution of 12 bits each, and two UART modules, 3 and 2 comparators $\mathrm{I} 2 \mathrm{C}$ modules. This component is connected to a Zigbee communication device using one of its UART modules. Its operating voltage is between $2.0 \mathrm{~V}$ and $5.5 \mathrm{~V}$, being compatible with the nominal voltage values necessary for a Zigbee device. This device can be configured to improve energy efficiency, with modes "Idle" and "Sleep" which can allow the outputs of the ports or internal devices such as watches, communication modules and A / D converters are ignored.

- Zigbee communication device: For communication from accumulators measures to the network coordinator, were chosen XBee devices, which are able to transmit at a rate of $250000 \mathrm{bps}$ and have a throughput of up to $35000 \mathrm{bps}$, enough capacity to transmit data small, as are the details of our network. Network support point-to-point technologies, point-to-multipoint and mesh.

Conditioners circuits: These circuits are variable depending on the sensor being used to monitor physical variables, can be composed of regulators, operational amplifiers, diodes and transistors court. For purposes of checking the energy consumption of a sensor node, technical documents of the elements are taken specifications.

Define the equation of current consumption for a node is defined by (2):

$$
I_{T}=I_{P}+I_{M}+I_{S}+I_{C}
$$

In which the total current $I_{T}$ is the sum of current consumption of all elements of the sensor node, with $I_{P}$ the power consumption by the processor, $I_{M}$ the power consumption by the storage modulus (Memory), $I_{S}$ consumption current by the sensor, and $I_{C}$ the power consumption by the communication module.

For our case, the storage module and the processor are the same device, and the sensor will RFID card reader. Our equation is modified as follows (3):

$$
I_{T(\tau)}=I_{P(\tau)}+I A_{S(t s)}+I I_{S(\tau-t s)}+I A_{C(t c)}+I A_{C(\tau-t c)}
$$

In $\tau$ defining the entire cycle time execution of the routine, and $t s, t m y$ tc define the time during which each element is in its active state, in the same form $E A$ it defines the current consumption of the item when it is active and $E I$ the current consumption the element when it is idle. Table I shows the values of energy consumption for the selected devices:

TABLE I: POWER CONSUMPTION OF THE DEVICES CHOSEN FOR THE DESIGN

\begin{tabular}{|l|l|l|}
\hline Device & Energy Tx & Energy Sleep \\
\hline $\begin{array}{l}\text { Micro-controler } \\
\text { PIC24FV32KA304 }\end{array}$ & $200 \mu \mathrm{A}$ & $10 \mu \mathrm{A}$ \\
\hline XBee S2B module & $117000 \mu \mathrm{A}$ & $15000 \mu \mathrm{A}$ \\
\hline RFID SL025M reader & $80000 \mu \mathrm{A}$ & $80000 \mu \mathrm{A}$ \\
\hline Total & $197200 \mu \mathrm{A}$ & $95010 \mu \mathrm{A}$ \\
\hline
\end{tabular}

For a cycle of one minute, two seconds of time calculated asset value in the XBee device performing transmission. The micro-controller, despite having different values for inactivity and activity, is always running, so its value will always be active. We can define then to a measurement cycle, the result is (4):

$$
\begin{aligned}
E_{T(\tau)}= & 200 \mu A \times 60 \mathrm{~s}+80000 \mu A \times 60 s+117000 \mu A \\
& \times 2 s+15000 \mu A \times 58=5916 m A
\end{aligned}
$$

Dividing by the cycle time, we have our total consumption $98 \mathrm{~mA}$ is accumulator measures .

- Network Coordinator: This device will consist of three main parts:

- Communication device Zigbee: Zigbee communication device have the same characteristics as described above measures the accumulator.

- Low-power computer: To perform data processing tasks, it has a low-cost computer called Raspberry Pi Model B.

This computer has a $700 \mathrm{MHz}$ processor, $256 \mathrm{MB}$ of memory and hard disk drives like an SD memory occupies a special port. This model also has 2 USB ports and an Ethernet port to allow network communication. In addition, you can connect the device with a high definition display through the HDMI port that owns the card. With a Linux operating system, called Raspbian. This computer allows developments with tools like Python, C, C ++ and Java. Allowing for the development to deliver the data to the Storage Server via IP frames, as well as viewing the data, if necessary.

- Reader RFID card and NFC: Due to the network also made identification processes, both personnel and inventory tests have been operating a RFID reader and NFC card connected to the computer saving Raspberry. For testing a card reader MIFARE SL025M (Fig. 1) he was chosen. Which supports Mifare 1K type tags, Mifare 4K NTAG203 MifareUltraLight and NFC. The handle two types of technologies, RFID and NFC, allowing also can use two types of tags, separating the RFID cards for identification processes of staff working or visiting the 
headquarters, and leaving the NFC tags for inventory items monitor as needed.

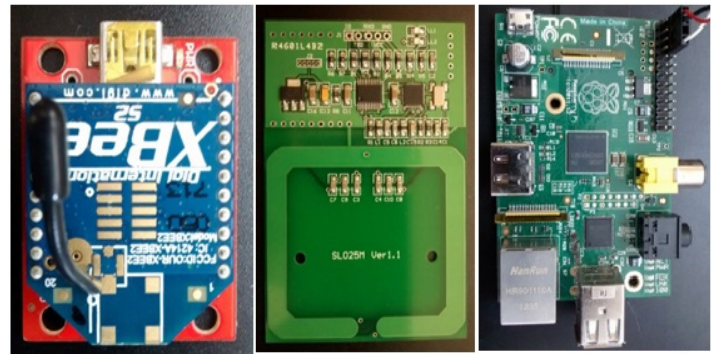

Fig. 1. Hardware wireless sensor network to testing.

- RFID tags of communication: In order to perform identification processes, both as a personal inventory building, deploying RFID tags or identification marks is necessary. These tags will be registered with RFID readers that are connected to computers with low power consumption and allow to know and record the entry and exit of personnel to each of the floors or special rooms. Mifare $1 \mathrm{~K}$ and Mifare $4 \mathrm{~K}$ cards are used.

- Communication NFC tags: You can also add tags NFC communication, for some elements who also want to be identified wirelessly, especially inventory items such as computer equipment and office. Due to restrictions of SL025M reader, NFC tags type NTAG203 be used. These tags come in the form of stickers are very easy to put any item in inventory.

- Storage server: This component is able to receive all data sent over the network coordinators, and store the data in a predefined database. This server must be able to provide access to data from different applications, so it must have features of Web server to deliver data through WS (Web Service) programmed in Java and also be able to show through a graphical interface stored data. Storage This server should use Linux operating system and have installed Java packages and MySQL development.

Due to the amount of data it can handle the storage server, it is suggested that high availability clusters, which address multiple requests for information are implemented. Additionally you must perform the "Sizing" of the database in order to determine what will be its total size, and how often should migrate legacy data to servers with less.

\section{Testing OF COMUniCATION}

To verify the network elements, tests Zigbee communication between the two modules used are made. The tests focused on setting the power of the messages sent and received, and lost frames as the distance increases between the points of emission and reception.

The test was performed with the modules connected through XCTU, connected to a computer and a laptop table respectively. Communication is at 19200 baud. Two environments have been taken for testing. One can be called as a test Indoor without direct line of sight, specifically away by a wall of glass blocks and home furnishings. Another test was conducted with the Coordinator on the 4th floor of an apartment building, and the router moving through the remaining floors of the building, always on the same horizontal period, varying only the vertical (top) between each measurement. To test the RSSI value, which for Xbee pro varies between -36 and $-100 \mathrm{dBm}$ sought, as it is shown in the following Table II:

TABLE II: RSSI READING LEVELS AT DIFFERENT DISTANCES
\begin{tabular}{|l|l|l|l|l|l|}
\hline $\begin{array}{l}\text { DIST } \\
(\mathrm{m})\end{array}$ & $\begin{array}{l}\text { RSSI } \\
(\mathrm{dBm})\end{array}$ & $\begin{array}{l}\text { DIST } \\
(\mathrm{m})\end{array}$ & $\begin{array}{l}\text { RSSI } \\
(\mathrm{dBm})\end{array}$ & $\begin{array}{l}\text { DIST } \\
(\mathrm{m})\end{array}$ & $\begin{array}{l}\text { RSSI } \\
(\mathrm{dBm})\end{array}$ \\
\hline 0.25 & -40 & 4.25 & -55 & 8.25 & -68 \\
\hline 0.50 & -40 & 4.50 & -55 & 8.50 & -70 \\
\hline 0.75 & -40 & 4.75 & -58 & 8.75 & -72 \\
\hline 1.00 & -41 & 5.00 & -57 & 9.00 & -72 \\
\hline 1.25 & -43 & 5.25 & -58 & 9.25 & -75 \\
\hline 1.50 & -43 & 5.50 & -60 & 9.50 & -78 \\
\hline 1.75 & -45 & 5.75 & -61 & 9.75 & -82 \\
\hline 2.00 & -45 & 6.00 & -59 & 10.0 & -80 \\
\hline 2.25 & -47 & 6.25 & -62 & 10.25 & -85 \\
\hline 2.50 & -46 & 6.50 & -63 & 10.5 & -89 \\
\hline 2.75 & -49 & 6.75 & -63 & 10.75 & -91 \\
\hline 3.00 & -49 & 7.00 & -63 & 11.00 & -90 \\
\hline 3.25 & -50 & 7.25 & -65 & 11.25 & -92 \\
\hline 3.50 & -52 & 7.50 & -65 & 11.50 & -94 \\
\hline 3.75 & -53 & 7.75 & -64 & 11.75 & -97 \\
\hline 4.00 & -53 & 8.00 & -67 & 12.00 & -98 \\
\hline
\end{tabular}

It can be concluded that with increasing distance, the RSSI decreases. And the longer the distance, the signals are becoming more random, due to the larger number of reflection losses. Complying with expectations for a wireless signal in free space. It defines the maximum distance covered in a indoor area is 12 meters, just as can be seen in Table III.

TABLE III: RSSI MEASSURENT LEVEL BETWEEN FLOOR

\begin{tabular}{|l|l|}
\hline FLOOR DISTANCE & RSSI $(\mathrm{dBm})$ \\
\hline 0 & -45 \\
\hline 1 & -62 \\
\hline 2 & -70 \\
\hline 3 & -80 \\
\hline 4 & -89 \\
\hline
\end{tabular}

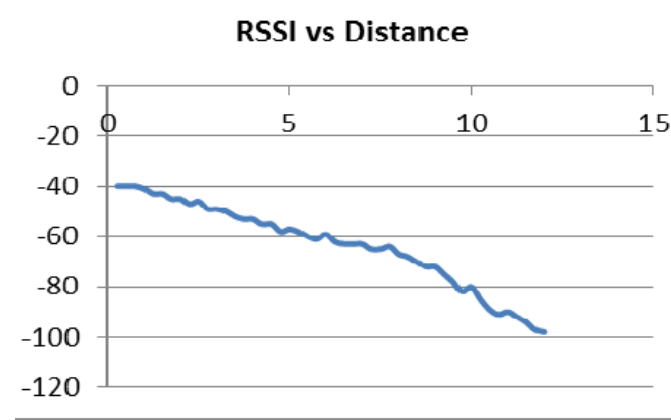

Fig. 2 a). RSSI testing graphics.

RSSI vs Floor

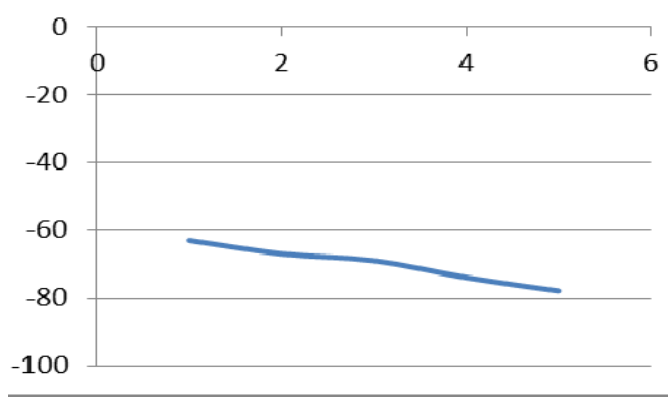

Fig. 2 b). RSSI testing graphics.

It can see in Fig. 2 a) and Fig. 2 b) that although the RSSI decreases according as the floors are increasing, we noticed a 
big difference between the signal down one floor after which additional floors down. Losing almost $20 \mathrm{dBm}$ in the first jump, then losing less than $10 \mathrm{dBm}$ in subsequent jumps.

\section{NETWORK DESIGN}

The distribution of sensor nodes is performed according to the coverage area, the transmission power and the amount of traffic that can be generated on each node. Due in this design variables are variables measured at slow changes, traffic is no problem since samples are taken and sent at a constant rate which is very low. However the coverage area and transmission power are factors to consider for design.

The coverage area is an indoor area, which establishes multiple transmitters losses due to having to walk through walls to carry the signal to a receiving point, which is why it has designed a distributed hierarchical design levels of the building. Additionally because of this environment, it is necessary to perform a-wireless fence, hybrid configuration to ensure proper data transport and total availability receptor level, since to them the identification systems are configured, they will be treated as part of the network of sensors.

The design has been made is a three-layer design (Fig. 3), in which you have a Storage Server, which obtains data from all recipients of level to which statement and stores is data from a database for later review. Receptor levels have access to all the sensor nodes level or floor on which are located, which will be referred to as accumulators, and which are connected directly to nodes and accumulate all measurements before transmitting a single frame. Recipients of experimental room, if needed, will be the fourth level in the hierarchy, monitoring all signals which may be generated in a particular room.

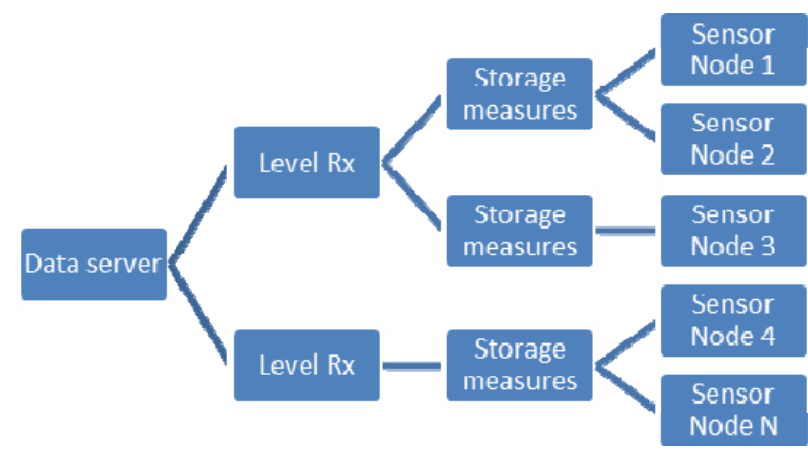

Fig. 3. Hierarchical WSN designed.

\section{A. Design of a Database for the Sensor Network and Storage}

Due to the network must be a configurable network, it is necessary that several values can be dynamically changed, so that the information is kept consistent. That is why, as defined multiple tables, which can get valuable information from the network and its components. Then the entity-relationship diagram (Fig. 4) of the database defined shown.

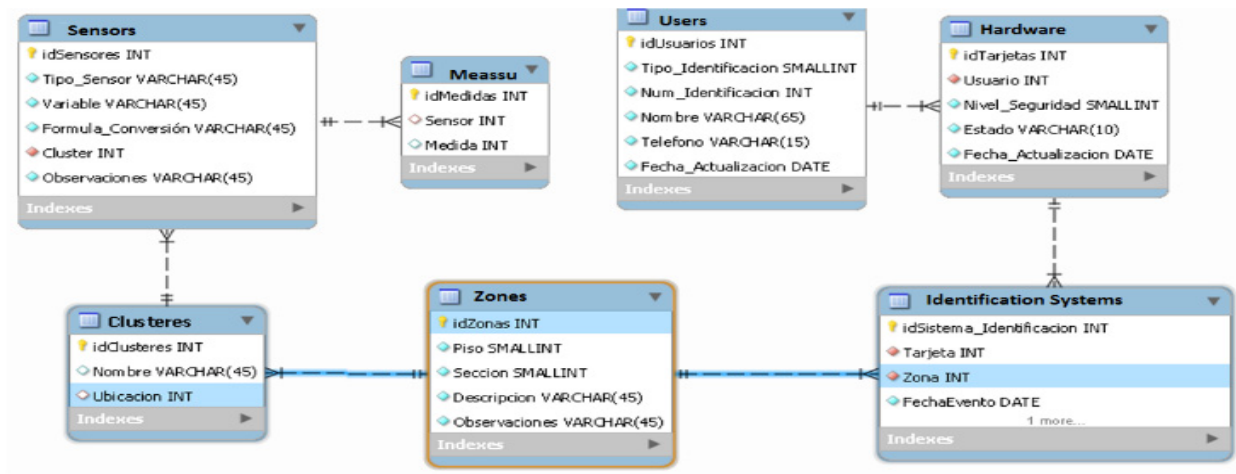

Fig. 4. WSN Entity-relationship database designed.

Sensor configuration table: the parameters of the sensors used in the network are set out in this table, the identifier, the type of sensor, the location, the type of variable we are measuring and geographical location is established within the building. Because all data is recorded through the A / D, but their actual values are different, the formula field will save the information necessary to convert the data collected in real value.

Configuration table for zones: In this table areas are established within the site of interest in which can be integrated sensors. They have an ID and a description.

Cluster configuration table: This table clusters formed within the network are set additionally each cluster can be associated to a specific area.

Configuration table ID card: This table shows the list of the RFID cards and users that were assigned, just as is done a security level defined for each card and have parameters to track.
User settings table: This table storing users entering the building is done, get up a record each time you get a new user. The user must register their personal details provided must be assigned an identification card in the configuration table ID card.

Tracking table system ID: This table tracking the activities of identification cards is done, each time a card is used a new event is generated, the event has a type (In, Out), a zone where it happened and date. This allows control over users who are inside the building.

Storage table measures: This table storing all measurements performed by the network, is a board composed only of four fields, and it is imperative that no wasted space. Each measurement should have a date, the identifier of the sensor that delivers the information, the measurement value and a serial number that is generated each time a field is entered in the table.

The database must be further fueled master tables 
encoding various elements like the types of identification users, types of card status, the types of levels of security and many other tables because they do not form a fundamental part of development, are not included in the diagram or design.

\section{NETWORK SERVICES}

The network designed, can be used to display different services within the research building (Fig. 5). In addition to the surveillance of the measured variables (temperature, humidity, light, rain, etc.) Can be used to design actuators make decisions from the measured data.

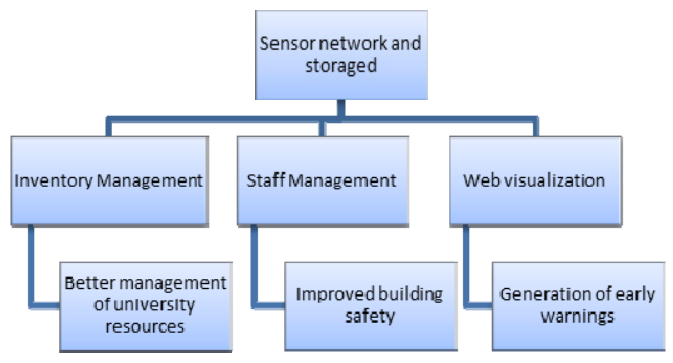

Fig. 5. Identification of services provided by the sensor network.

\section{A. Generation and Shaping of the Zigbee Frame}

Communication between measurement and accumulators coordinators Power is delivered via Zigbee modules, so it is necessary to define how this communication takes place at the level of plot. For each sensor 4 bytes of information are used, the first two bytes contain the identification of the sensor, while the other 2 bytes are used to deliver information from the sensor measurement. Since not all measures accumulators have the same number of connected sensors, the frame length is variable, using 2 bytes to define how much information (in bytes) of each frame.

The formation of the plot will be made on a routine micro-controller Table IV), which will verify their ports $A / D$, it will store the information obtained and stored according to the defined protocol.

TABLE IV: FORMATION OF THE PLOT OF ZIGBEE DATA

\begin{tabular}{|l|l|l|l|}
\hline SINCRONIZATION & $\begin{array}{l}\text { LONG } \\
\text { FRAME }\end{array}$ & DATE & CHECKSUM \\
\hline 2 Bytes & 2 Bytes & N Bytes & N Bytes \\
\hline
\end{tabular}

Finally, the complete network design, shown in the following Fig. 6:

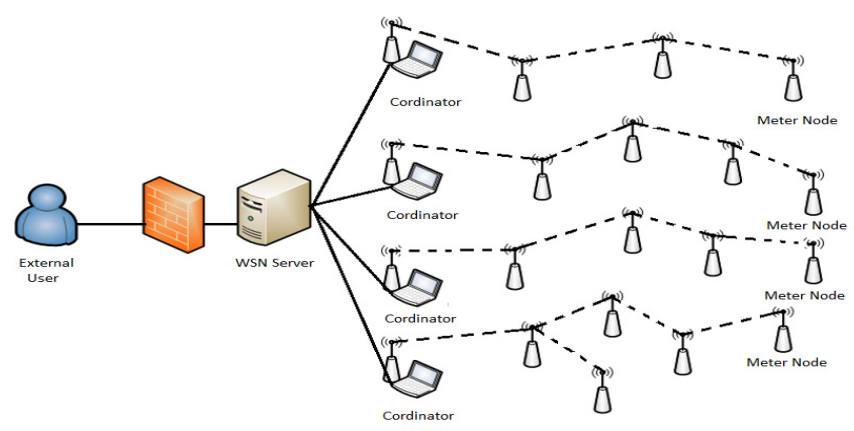

Fig. 6. Diagram of the network of sensors designed.

\section{CONCLUSIONS}

The design of a network of sensors and scalable deployment, ZigBee and RFID-based research building for the University FJC District was conducted, however, the design can be applied to any building or closed room of the same characteristics.

Including a configurable database allows the inclusion of new sensors easier, also allowing the data to be sent directly as they take the A / D conversions for obtaining data with requested metrics, it is done when consulting the database, by managing the formula field.

RFID sensors allow the network to keep track of objects that are in the inventory of the university, as well as personnel entering into the building.

It is proposed as future work, the creation of visualization system data network of sensors.

The authors are currently working on finding a way to make broadcasts events when getting changes in the measured variables.

\section{REFERENCES}

[1] R. G. Vieira, A. M. Cunha, and F. F. Silveira, "A WSN energy management architecture applied to the Amazonian region," in Proc. 7th International Conference on Information Technology, 2010, pp. 1159-1164.

[2] D. Baghyalakshmi, J. Ebenezer, and S. A. V. Satyamurty, "WSN based temperature monitoring for high performance computing cluster," in Proc. International Conference on Recent Trends in Information Technology, 2011, pp. 1105-1110.

[3] D.-Z. Sun, S. Jiang, W.-X. Wang, and J.-C. Tang, "WSN design and implementation in a tea plantation for drought monitoring," in Proc. International Conference on Cyber-Enabled Distributed Computing and Knowledge Discovery, 2010, pp. 156-159.

[4] R. D. Pietro et al., "Energy efficient node-to-node authentication and communication confidentiality in wireless sensor networks," Wireless Networks, vol. 6, no. 6, 2006, pp 709-721.

[5] L. M. Kamarudin, R. B. Ahmad, B. L. Ong, A. Zakaria, and D. Ndzi, "Modeling and simulation of near-earth wireless sensor networks for agriculture based application using OMNeT++," in Proc. International Conference on Computer Applications and Industrial Electronics, 2010, pp. 131-136.

[6] P.-P. Chen and Z.-M. Lu, "A web-based indoor environment monitoring system using wireless sensor networks," in Proc. International Conference on Computational and Information Sciencies, 2013, pp. 2007-2010.

[7] Z.-Z. Wu, Z.-L. Liu, J. Liu, and X.-W. Huang, "Wireless sensor networks for living environment monitoring," in Proc. World Congress on Software Engineering, 2009, pp. 22-25.

[8] P. Spachos, S. Liang, and D. Hatzinakos, "Prototypes of opportunistic wireless sensor networks supporting indoor air quality monitoring," in Proc. 10th Annual IEEE Consumer Communications and Networking Conference, 2013, pp. 851-852.

[9] G. Stamatescu and V. Sgarciu, "Evaluation of wireless sensor network monitoring for indoor spaces," in Proc. International Symposium on Instrumentation and Measurement, Sensor Network and Automation (IMSNA), 2012, pp. 107-111.

[10] X.-Y. Cui, X.-D. Zhang, and Y.-K. Shang, "Energy-saving strategies of wireless sensor networks," in Proc. 2007 International Symposium on Microwave, Antenna, Propagation, and EMC Technologies For Wireless Communications, 2007, pp. 178-181.

[11] X.-F. Liu, J.-N. Cao, S. Lai, C. Yang, H.-J. Wu, and Y.-L. Xu, "Energy efficient clustering for WSN-based structural health monitoring," in Proc. IEEE INFOCON, 2011, pp. 2768-2776.

[12] J.-Y. Wang, C.-P. Chen, T.-S. Lin, C.-L. Chuang, T.-Y. Lai, and J.-A. Jiang, "High-precision RSSI-based indoor localization using a transmission power adjustement strategy for wireless sensor networks," in Proc. 14th IEEE Conference in High Performance Computing and Communications, 2014, pp. 1634-1638.

[13] T.-P. Huang, H.-M. Chen, Z.-L. Z, and L. Cui, "EasiPLEAD: Discriminating the causes of packet losses and errors in indoor WSN," in Proc. Ad Hoc an Sensor Network Symposium, 2012, pp. 487-493. 
[14] C. Walravens and W. Dehaene, "Design of a low-energy data processing architecture for WSN nodes," in Proc. Design, Automation and Test in Europe Conference, 2012, pp. 570-573.

[15] O. G. Adewumi, K. Djouani, and A. M. Kurien, "RSSI based indoor and outdoor distance estimation for localization in WSN," in Proc. 2013 IEEE International Conference on Industrial Technology (ICIT), 2013, pp. 1534-1539.

[16] F. Salim, M. Williams, N. Sony, M. Dela Pena, Y. Petrov, A. A. Saad, and B. Wu, "Visualization of wireless sensor networks using Zigbee's Received Signal Strength Indicator (RSSI) for indoor localization and tracking," in Proc. IEEE International Conference on Pervasive Computing and Communications Workshops (PERCOM Workshops), 2014, pp. 575-580.

[17] C.-H. Chu, C.-H. Wang, and C.-K. Liang, "High-accuracy indoor personnel tracking system with a ZigBee wireless sensor network," in Proc. 7th International Conference on Mobile Ad-Hoc and Sensor Networks, 2011, pp. 398-402.

[18] Z.-B. Xiong, F. Sottile, M. A. Spirito, and R. Garello, "Hybrid indoor positioning approaches based on WSN and RFID," in Proc. 2011 4th IFIP International Conference on New Technologies, Mobility and Security (NTMS), 2013, pp. 1-5.

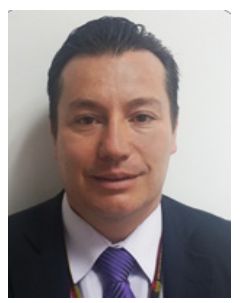

Roberto Ferro Escobar was born in Bogotá, Colombia. He received the $\mathrm{PhD}$ degree in informatic engineering from the Pontificia of Salamanca University, the master in tele-informatic and electronic engineering of the District University "Francisco José de Caldas" of Colombia. He is currently a researcher and a senior lecturer at the Faculty of Engineering at the District University "Francisco José de Caldas" in Bogotá, Colombia, where he is the dean of Engineering Faculty. He was the director of engineering doctorate. $\mathrm{He}$ is also a professor in UNIR - International
University of the Rioja. His area of work and research is networking, electronic, TIC, aero special technology and software.

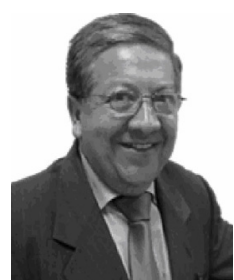

Víctor Hugo Medina García was born in Santa Rosa de Viterbo, Colombia. He received the $\mathrm{PhD}$ degree in computer engineering from the Pontificia of Salamanca University, DEA Languages, Systems and Software Engineering and Master in Computer Science at the Politécnica University of Madrid in Spain, a specialist in marketing from the Rosario University and Systems Engineering of the Distrital University "Francisco José de Caldas" of Colombia. He is currently a researcher and a senior lecturer at the Faculty of Engineering at the Distrital University "Francisco José de Caldas" in Bogotá - Colombia, where he is an extension director of Engineering Faculty, he was the director of engineering doctorate. He is also a professor in UNIR - International University of the Rioja and he was a coordinator, an associate professor and a visiting professor in computer engineering from the Pontificia of Salamanca University Campus of Madrid, Spain. His area of work and research is knowledge management and software engineering.

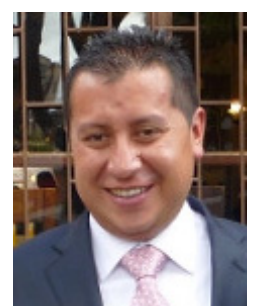

Giovanny Tarazona Bermudez was born in Bogotá, Colombia. He received the $\mathrm{PhD}$ degree in engineering from the Oviedo of University, a specialist in software engineering from the District University and Industrial Engineering of the District University "Francisco José de Caldas" of Colombia $\mathrm{He}$ is currently a researcher and a senior lecturer at the Faculty of Engineering at the District University "Francisco José de Caldas" in Bogotá, Colombia, where he is the director of engineering doctorate. And he was the dean of engineering. His area of work and research is project management. 